\title{
CONSEQÜÊNCIAS DA LAMINECTOMIA DORSAL DO TIPO FUNKQUIST A EM CÃES NORMAIS
}

\author{
CONSEQUENCES OF THE FUNKQUIST A DORSAL \\ LAMINECTOMY, IN NORMAL DOGS
}

\author{
Eduardo Alberto Tudury ${ }^{1}$ Sheila Canevese Rahal ${ }^{2}$ \\ Dominguita Lühers Graça $^{3}$ Jamile Neto Haddad ${ }^{4}$ \\ Mônica Vicky Bahr Arias ${ }^{4}$ Otávio Pedro Neto ${ }^{5}$
}

\section{RESUMO}

$O$ objetivo desta pesquisa foi verificar os efeitos da laminectomia dorsal do tipo Funkquist A em cães normais. A técnica realizada consistiu em abordagem dorsal à coluna vertebral; remoção (com ou sem a ajuda da elevação dos corpos vertebrais) das lâminas dorsais de $T_{13}$ e $L_{1}$; manutenção do ligamento supra-espinhal; colocação de gordura subcutânea como coxim supramedular; sutura da fáscia toracolombar, subcutâneo e pele; e bandagem compressiva por sete dias. Quarenta e oito horas após a cirurgia, todos os 10 cães submetidos a essa técnica exibiram perda das reações posturais e paresia de moderada a grave nos membros pélvicos. Hipoalgesia foi constatada em cinco deles. Tentando descobrir as causas, foram realizados estudos histológicos de medulas espinhais, coletadas 4 e 48 horas após a realização da laminectomia, porém com modificações como: não elevação dos corpos vertebrais durante a cirurgia e prevenção de qualquer tipo de compressão medular após a laminectomia. Analisando esses resultados, foi possível concluir que as disfunções neurológicas decorreram de lesões medulares ocasionadas possivelmente por: elevações dos corpos vertebrais durante a laminectomia, danificação de vasos espinhais, desestabilização da coluna vertebral e uma somatória de forcas compressivas atuantes sobre a medula espinhal desprotegida. Na necropsia de cinco cães, acompanhados por quarenta e cinco dias, foi possível constatar: a permanência do ligamento supra-espinhal; que o enxerto de gordura não evita a penetração do tecido fibroso no canal vertebral nem sua aderência à duramáter; notório $(P<0.01)$ achatamento do canal vertebral $e$ deformação das medulas espinhais. Com base nos resultados, aconselha-se não realizar esses procedimentos cirúrgicos em cães acometidos de afeções medulares na junção toracolombar. A manutenção do ligamento supra-espinhal não perturba a realiza- ção da laminectomia, minimiza a ocorrência de defeitos estéticos na linha média dorsal e pode participar da compressão medular no pós-operatório imediato.

Palavras-chave: coluna vertebral, cirurgia de cães, laminectomia.

\section{SUMMARY}

The purpose of this research was to check the effects of the Funkquist A dorsal laminectomy in normal dogs. The technique accomplished consisted in dorsal approach to the spine; removal of $T_{13}-L_{1}$ laminae with or without vertebral bodies elevation; mantainement of the supraspinous ligament; placement of subcutaneous fat as supramedulary pad; suture of thoracolumbar fascia, subcutaneous and skin; and compressive bandage for seven days. Fourty-eight hours latter all the dogs (10) submitted to this technique showed loss of the postural reactions and paraparesis classified of moderated to serious. Hypalgesia was ascertained in five of them. Trying to discover the causes, was accomplished histologic studies of spinal cord collected 4 and 48 hours after the accomplishment of the laminectomies were conducted, however with modifications as: non-elevation of the vertebral bodies during the surgery and prevention of any type of compression spinal after the laminectomie. Examining these results was possible to conclude that the neurologic dysfunctions were originated from cord lesions caused for: elevation of the vertebral bodies during the laminectomy, possible damage of spinal vessels, destabilization of the spine and a somatory of compressive forces acting upon an uncovered spinal cord. At the necropsy of five dogs realized forty - five days latter were possible to verify: the permanence of the

\footnotetext{
${ }^{1}$ Médico Veterinário, Doutor, Professor Adjunto 1, Departamento de Medicina Veterinária, Universidade Federal Rural de Pernambuco, (UFRPE). Rua Dom Manoel de Medeiros s/n, Dois Irmãos, 52171-900. Recife, PE. E-mail: tudury@ @elore.npde.ufrpe.br. Autor para correspondência.

${ }^{2}$ Médico Veterinário, Doutor, Professor adjunto, Faculdade de Medicina Veterinária e Zootecnia, UNESP, Botucatu - SP.

${ }^{3}$ Médico Veterinário, PhD., Professor Titular, Departamento de Patologia (DP), Universidade Federal de Santa Maria (UFSM), 97105900, Santa Maria, RS.

${ }^{4}$ Médico Veterinário, Mestre, Professor Assistente, Universidade Estadual de Londrina, Londrina, PR.

${ }^{5}$ Médico Veterinário, aluno do curso de Pós-graduação da UFRPE.
} 
supraspinous ligament; that the fat grafts do not avoid the penetration of the fibrous tissue into the vertebral canal neither its adherency to the dura mater; notorious $(p<0.01)$ flattening of the vertebral canal and deformations of the spinal cords. With support on these results, isn't advise to furfill these surgery procedure in the thoracolumbar junction of dogs. The mantainement of the supraspinatus ligament do not perturb the realization of the laminectomy, minimize the occurrence of the aesthetics defects at the dorsal midline and can participate at the immediate postoperative cord compression.

Key words: spine, surgery of dogs, laminectomy.

\section{INTRODUÇÃO}

A cirurgia vertebral e de medula espinhal em cães e gatos é baseada em três procedimentos: descompressão, fixação e exploração (SWAIM, 1987; SMITH \& WALTER, 1988). A descompressão da medula espinhal é acompanhada pela remoção de parte do arco vertebral e de materiais que ocupam espaço no canal vertebral (GAMBARDELLA, 1980; SWAIM, 1987; YOVICH et al., 1994). As técnicas descompressivas para a coluna toracolombar compreendem as hemilaminectomias (unilaterais e bilaterais), minihemilaminectomias e laminectomias dorsais (SWAIM \& VANDEVELDE, 1974; TROTTER $\boldsymbol{e t}$ al., 1975; HORNE et al., 1977; SMITH \& WALTER, 1988; YOVICH et al., 1994), sendo que essas últimas apresentam-se em quatro modalidades: laminectomias dorsais dos tipos Funkquist A e B, laminectomia dorsal modificada e laminectomia dorsal profunda. (SWAIM \& VANDEVELDE, 1974; TUDURY et al., 1985; SWAIM, 1987).

A laminectomia do tipo Funkquist A é caracterizada pela excisão dos arcos vertebrais e processos articulares em um nível discretamente inferior à superfície da medula espinhal. (FUNKQUIST, 1962; FUNKQUIST \& SCHANTZ, 1962; SWAIM \& VANDEVELDE, 1974; TROTTER et al., 1975; HORNE et al., 1977). O inconveniente dessa técnica é que, durante a formação e maturação da membrana da laminectomia, ocorre compressão secundária da medula espinhal contra o assoalho do canal vertebral (LOROCCA \& MACNAB, 1974; TROTTER et al., 1975; TROTTER et al., 1988; TROTTER, 1990). Os principais déficits neurológicos decorrentes da laminectomia do tipo Funkquist A são cifose, variados graus de ataxia-paresia e paralisia, (FUNKQUIST \& SCHANTZ, 1962; FIALHO \& BERNIS, 1975). Em estudos prévios, cifose foi observada em $16(59,2 \%)$ de 27 cães submetidos a essa cirurgia, enquanto que também foi observada paresia durante as primeiras semanas e meses, após melhora temporária dos animais (FUNKQUIST \& SCHANTZ, 1962; HORNE et al., 1977). A sutura da fáscia toracolombar pare- ceu contribuir com paresia durante a primeira semana do pós-operatório em quatro de nove animais (FUNKQUIST \& SCHANTZ, 1962). Quando os processos articulares ou quando os ligamentos supra-espinhoso e interespinhoso são removidos, ocorre diminuição de $75 \%$ da estabilidade vertebral (FIALHO \& BERNIS, 1975; SMITH \& WALTER, 1988). Desa maneira, a laminectomia descompressiva torna o paciente mais suscetível a lesões medulares traumáticas pós-operatórias (SMITH \& WALTER, 1988).

Nos locais da laminectomia Funkquist A, macroscopicamente, observam-se vários graus de compressão da medula espinhal (FUNKQUIST, 1962; FUNKQUIST \& SCHANTZ, 1962; TROTTER $\boldsymbol{e t}$ al., 1975), acompanhados microscopicamente de lesões típicas de compressão como degeneração difusa em todos os funículos, substância branca com aparência espongiforme e diminuição dos neurônios na substância cinzenta. (TROTTER $\boldsymbol{e} t$ $\boldsymbol{a l}$., 1975). Entretanto, em outras técnicas de laminectomia em cães, a fibrose constritiva não causa problemas significantes (FUNKQUIST, 1970; HORNE et al., 1977; TROTTER, 1990), sendo que a constrição da medula espinhal pela membrana da laminectomia é influenciada pela extensão da descompressão e pelo nível atingido na ressecção dos pedículos vertebrais. (FUNKQUIST, 1962; LOROCCA \& MACNAB, 1974; SWAIM \& VANDEVELDE, 1974; HORNE et al., 1977; SWAIM, 1987). Além disso, as investigações sugerem causas multifatoriais para a membrana da laminectomia como, por exemplo, localização anatômica específica da cirurgia na coluna vertebral; métodos e adequação da hemostasia; extensão da gordura epidural removida; o tipo, orientação e colocação exata de vários implantes dorsais, especialmente quanto ao seu encaixe marginal (TROTTER et al., 1988; TROTTER, 1990; TREVOR et al., 1991).

Implantes biológicos e não biológicos permanentes ou biodegradáveis têm sido colocados em diferentes orientações e configurações procurando fornecer uma barreira perfeita contra a invasão de tecido fibroso no espaço epidural dorsal (TROTTER et al., 1988; TREVOR et al., 1991). Em estudos experimentais e clínicos, a gordura autógena interposta entre a dura-máter e a musculatura paraespinhal, ao contrário das membranas não biológicas, serviu como uma barreira efetiva limitando o crescimento de tecido conjuntivo dentro do canal espinhal (KELLER $\boldsymbol{e t}$ al., 1978; TROTTER $\boldsymbol{e t}$ al., 1988; TREVOR et al., 1991). Esta função é favorecida pela sua flexibilidade, proporcionando perfeito encaixe às margens do local da laminectomia e preve- 
nindo fendas, ao longo das quais o tecido fibroso possa invadir (TREVOR et al., 1991). Embora seja reduzida no tamanho, a gordura autógena é revascularizada e sobrevive (KELLER et al., 1978; TREVOR et al., 1991).

O objetivo deste trabalho foi verificar os efeitos pós-operatórios (junto a suas respectivas causas) da laminectomia dorsal do tipo Funkquist A em cães normais.

\section{MATERIAL E MÉTODOS}

Neste estudo, foram utilizados 26 cães adultos de ambos os sexos, sem raça definida, pesando entre 4,5 e $14,4 \mathrm{~kg}$ (média de $8,11 \mathrm{~kg}$ ). Após exames clínicos e neurológicos, pesagem e tricotomia da região dorsal, os animais foram submetidos a um regime anestésico composto por sulfato de atropina $(0,04 \mathrm{mg} / \mathrm{kg} \mathrm{IM})$, cloridrato de xilazina $(1 \mathrm{mg} / \mathrm{kg}$ IM) e tiopental sódico para a indução e manutenção (aproximadamente 12,5mg/kg IV).

A técnica realizada foi a laminectomia do tipo Funkquist A com remoção das partes dorsal e dorsolateral do arco vertebral até o nível da tangente dorsal da medula espinhal, incluindo os processos articulares craniais e caudais de $\mathrm{T}_{13}$ e $\mathrm{L}_{1}$. Para melhor interpretação dos resultados, os cães foram distribuídos em cinco grupos e submetidos a cirurgias com variações, especificadas a seguir:

grupo I: composto por 7 cães, teve como objetivo verificar os efeitos gerais imediatos e tardios da técnica. Os animais foram submetidos à laminectomia facilitada pela elevação dos corpos vertebrais, colocação de gordura (retirada da própria área cirúrgica) como coxim supramedular, sutura mediante fio de náilon da fáscia toracolombar, subcutâneo e pele e bandagem compressiva por 7 dias. O período de observação foi de 45 dias quando, então, procedeuse a eutanásia de cinco animais, seguida da realização de mielografia e exame anatomopatológico;

grupo II: utilizaram-se 3 cães com o propósito de verificar se a técnica realizada no grupo I, agora, sem elevação dos corpos vertebrais, poderia gerar lesões medulares nas primeiras 48 horas, as quais foram verificadas através de exames clíniconeurológico e histopatológico de todos os animais; grupo III: formado por 3 cães, com o propósito de observar os efeitos imediatos da elevação dos corpos vertebrais durante a realização da laminectomia dorsal. Essa elevação foi realizada prendendo-se com pinças Backhaus os processos espinhosos das lâminas a serem removidas visando, assim, a uma separação tal dos processos articulares que facilitasse sua ressecção. Após a laminectomia, não foi colocada gordura subcutânea, suturas ou qualquer procedimento que pudesse promover compressão dorsal da medula espinhal. Após hidratação desta por 4 horas com solução fisiológica em temperatura ambiente, todos os animais foram submetidos à eutanásia para exames histopatológicos;

grupo $I V$ : formado por 3 cães, com o objetivo de verificar se apenas a remoção da lâmina vertebral causa algum dano imediato na medula espinhal. Elevação dos corpos vertebrais, colocação de gordura, como coxim supramedular e suturas, não foram realizados. A medula foi hidratada por 4 horas, até a eutanásia de todos os animais para exames histopatológicos;

grupo $V$ (controle): composto por 10 cães não operados e submetidos à eutanásia, teve como propósito fornecer medulas espinhais e colunas vertebrais normais para comparações quantitativas e/ou qualitativas com os grupos anteriores.

Os animais do grupo I, imediatamente após a eutanásia, foram submetidos à mielografia com o contraste iopamidol, injetado através da cisterna magna, com o intuito de detectar compressões do parênquima espinhal secundários à membrana da laminectomia. Ao exame de necropsia do mesmo grupo, buscou-se observar alterações como: presença de ligamento supra-espinhal, aderência de duramáter à membrana cicatricial, elevação da duramáter pela aderência fibrótica, achatamentos da medula espinhal e do canal vertebral, infiltração de tecido fibroso no canal vertebral, dilatação dos seios venosos e, também, ossificação cicatricial da membrana da laminectomia. Posteriormente, suas medulas foram enviadas para o setor de Patologia Veterinária da Universidade Federal de Santa Maria.

Além disso, as regiões operadas da coluna vertebral dos animais dos grupos I e V (controle) foram comparadas estatisticamente com base nas seguintes relações: (I) quociente entre os diâmetros verticais da medula espinhal e do corpo vertebral; (II) quociente entre os diâmetros vertical e horizontal da medula espinhal; (III) quociente entre os diâmetros verticais do canal e do corpo vertebral; (IV) quociente entre os diâmetros vertical e horizontal do canal vertebral. As medidas destas variáveis quantitativas foram realizadas com paquímetro, após fixação das amostras e secção transversal na região de disco intervertebral $\mathrm{T}_{13}-\mathrm{L}_{1}$. A análise estatística destas relações foram realizadas mediante esquema de comparação de pares, utilizando-se o teste "T" de Student para a comparação das médias (SNEDECOR \& COCKRAN, 1989).

\section{RESULTADOS}

Os cães de no 1 e 4, pertencentes ao grupo I, foram excluídos das análises estatísticas devido a abscesso e trauma medular transcirúrgico respecti- 
vamente. As alterações neurológicas dos cinco animais restantes pertencentes ao grupo I, observadas nas primeiras 48 horas e 45 dias pós-cirurgia, estão resumidas na tabela 1. Após recuperação anestésica, todos esses cães exibiram perda das reações posturais e paraparesia de moderada a grave, sendo que apenas quatro delas demonstraram retenção urinária e dois hipoalgesia. Cifose de grau leve a moderado esteve presente em quatro animais desse grupo. Nos cães do grupo II, as duas únicas alterações observadas nas primeiras 48 horas pós-cirurgia foram perda de reações posturais e paraparesia de moderada a grave.

Dos cinco cães do grupo I, acompanhados neurologicamente durante 45 dias (tabela 1), quatro deles apresentaram melhora das disfunções proprioceptivas, nociceptivas e motoras, inclusive, das disfunções vesicais. Enquanto a cifose se manteve estável ou progrediu, quatro dos cinco animais apresentaram depressão da linha média dorsal pela ausência dos processos espinhosos. À mielografia, observou-se estreitamento latero-lateral da medula espinhal na região dos discos intervertebrais $\mathrm{T}_{13}-\mathrm{L}_{1}$. As alterações observadas durante a necropsia, nesses mesmos animais do grupo I, estão relacionadas na tabela 2. Enquanto que o estreitamento do canal vertebral foi constatado em todos os animais necropsiados, foi observado em três cães notório achatamento da medula espinhal (Figura 1). Todos os animais tiveram aderência da dura-máter ao tecido cicatricial. Infiltração de tecido fibroso no canal vertebral, bem como dilatação dos seios venosos foram observados na maioria dos casos analisados (Tabela 2).

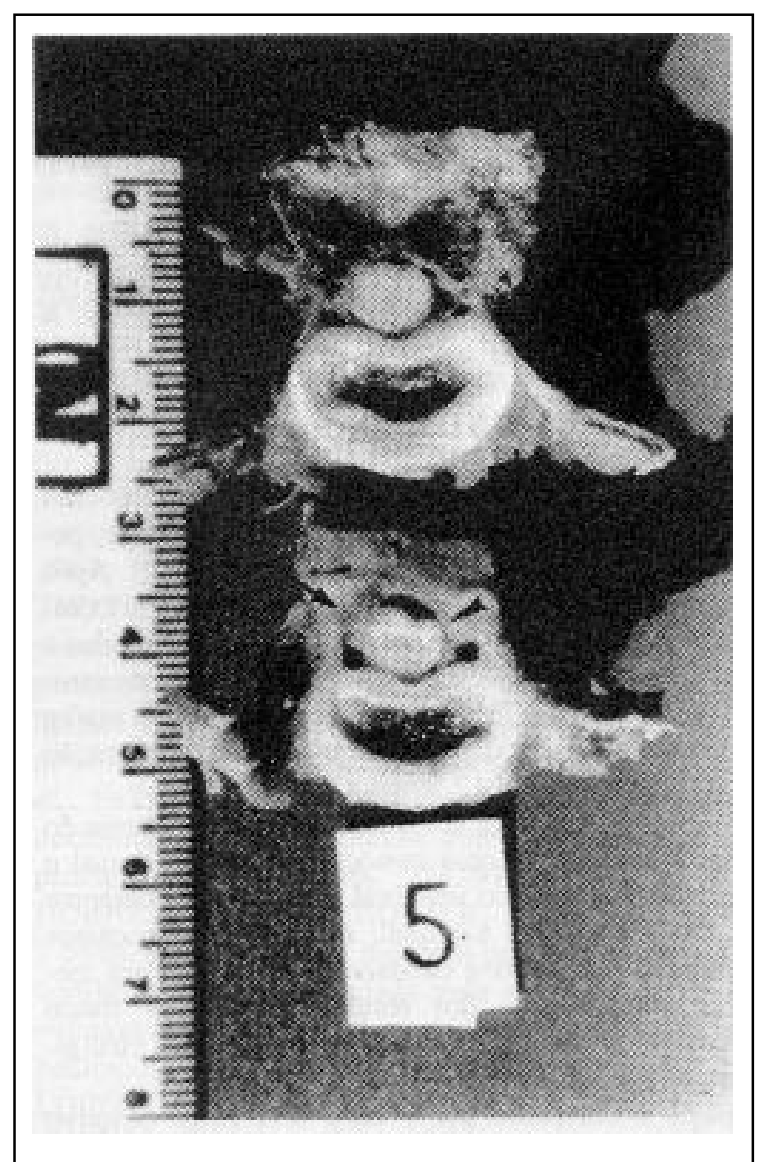

Figura 1 - Infiltração de tecido fibroso no canal vertebral (seta fina) e deformação da medula espinhal (cabeça de seta), observadas 45 dias após a laminectomia dorsal.

Tabela 1 - Alterações clínicas observadas nos cães do grupo I, 48 horas e 45 dias pós-laminectomia tipo Funkquist A.

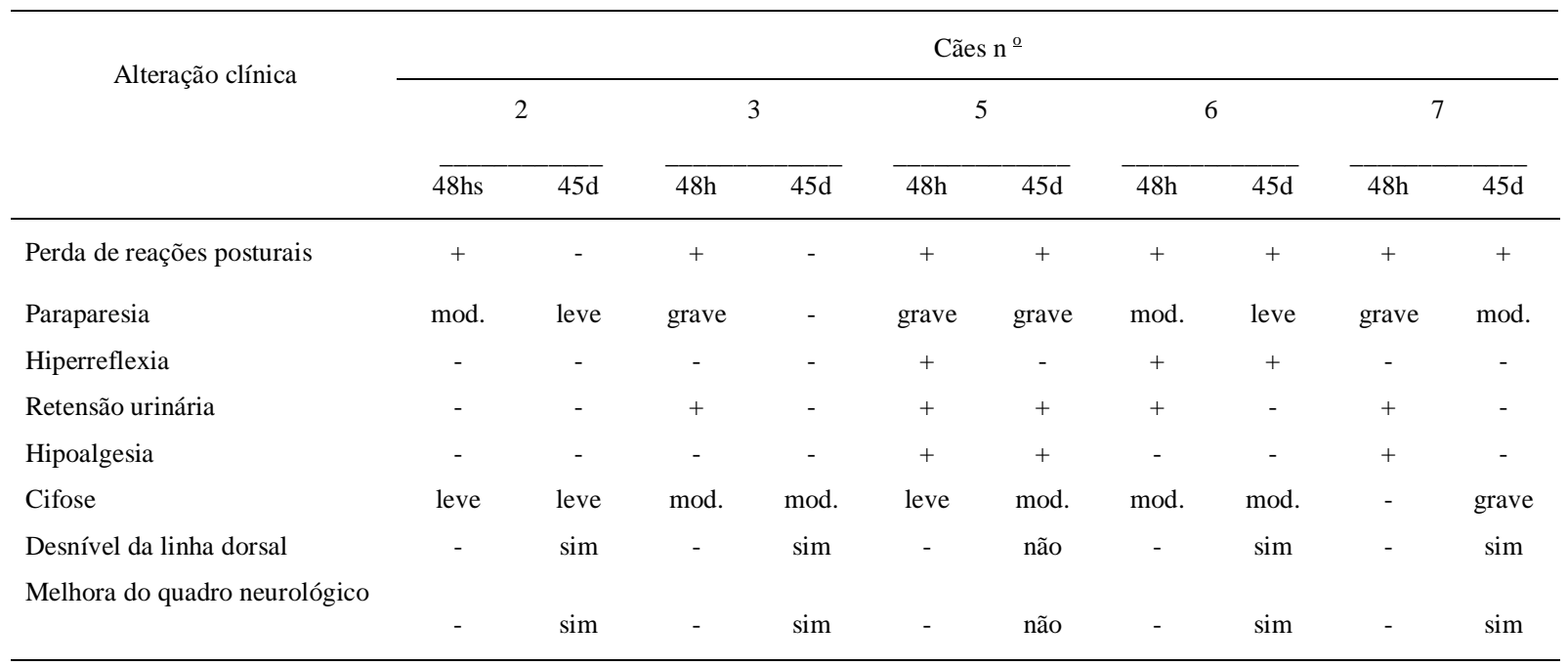

+ presença da alteração; - ausência de alteração; mod. = moderada. 
Tabela 2 - Achados de necropsia observados nos cães do grupo I, 45 dias pós-laminectomia tipo Funkquist A.
(FUNKQUIST \& SCHANTZ, 1962; FUN-KQUIST, 1962; FIALHO \& BERNIS, 1975; HORNE et al., 1977). Contudo, no presente estudo experimental, semelhante ao relatado por TREVOR $\boldsymbol{e t}$ al. (1991) em um estudo com laminectomia dorsal modificada, os sinais neurológicos ocorreram poucas horas após a cirurgia. Essa deterioração aguda, gerada pela laminectomia do tipo Funkquist A, pode ter ficado encoberta nos cães operados por FUNKQUIST (1962), pelas disfunções neurológicas já existentes antes da cirurgia. O "status" neurológico dos animais da presente pesquisa (paraparesia não ambulatorial,

O exame histopatológico revelou degeneração Walleriana, retração neuronal, processo astrocitário com discreta gliose, escassos balões e alguns ovóides com células gitter nos animais do grupo I; retração neuronal com infiltrado celular inflamatório, congestão da substância cinzenta, escassos balões e alguns ovóides no grupo II; degeneração Walleriana e sinais de isquemia neuronal no grupo III; sinais de isquemia, congestão da substância cinzenta e raros ovóides no grupo IV, sendo que, no grupo $\mathrm{V}$, observaram-se retração neuronal e congestão da substância cinzenta. A análise estatística comparativa dos animais dos grupos I e V (Tabela 3) revelou diferenças estatísticas significativas na região de $\mathrm{T}_{13}-\mathrm{L}_{1}$, quanto aos quocientes entre os diâmetros verticais do canal e do corpo vertebral $(\mathrm{p}<0.05)$ e, quanto aos quocientes entre os diâmetros vertical e horizontal do canal vertebral $(\mathrm{p}<0.01)$.

\section{DISCUSSÃO E CONCLUSÃO}

Conforme exposto na introdução, a laminectomia do tipo Funkquist A apresenta consequiências indesejáveis no pós-operatório quando comparadas às outras técnicas de laminectomia (FUNKQUIST \& SCHANTZ, 1962). Em estudos prévios, os déficits neurológicos secundários a estas técnicas foram observados principalmente nas primeiras semanas e meses, após melhora temporária das disfunções pré-cirúrgicas
Tabela 3 - Valor médio, desvio-padrão e resultado da análise estatística comparativa das medições realizadas nas medulas espinhais e vértebras (segmento $\mathrm{T}_{13^{-}}$ $\mathrm{L}_{1}$ ) dos animais dos grupos $\mathrm{I}$ e $\mathrm{V}$.

\begin{tabular}{lcc}
\hline \multicolumn{1}{c}{ Relação = tipo de quociente } & Grupo I & Grupo V \\
& $\overline{\mathrm{X} \pm \mathrm{s}^{*}}$ & $\overline{\mathrm{X}} \pm \mathrm{s}$ \\
\hline $\begin{array}{l}\text { I = diâmetros verticais da medula espinhal e } \\
\text { do corpo vertebral }\end{array}$ & $0,454^{\mathrm{a}} \pm 0,042$ & $0,513^{\mathrm{a}} \pm 0,071$ \\
$\begin{array}{l}\text { II = diâmetros vertical e horizontal da medula } \\
\text { espinhal }\end{array}$ & $0,741^{\mathrm{a}} \pm 0,033$ & $0,798^{\mathrm{a}} \pm 0,074$ \\
$\begin{array}{l}\text { III = diâmetros verticais do canal e do corpo } \\
\text { vertebral }\end{array}$ & $0,560^{\mathrm{a}} \pm 0,074$ & $0,701^{\mathrm{b}} \pm 0,108$ \\
$\begin{array}{l}\text { IV = diâmetros vertical e horizontal do canal } \\
\text { vertebral }\end{array}$ & $0,458^{\mathrm{a}} \pm 0,059$ & $0,639^{\mathrm{c}} \pm 0,085$
\end{tabular}

* X: média; s: desvio-padrão; as médias da mesma linha, seguidas por letras desiguais, diferem estatisticamente entre si (a-b: $\mathrm{P}<0,05$; $\mathrm{a}-\mathrm{c}$ : $\mathrm{P}<0,01)$. 
corpos vertebrais durante a laminectomia, possível danificação dos vasos espinhais durante o acesso cirúrgico e desestabilização da coluna vertebral, já que a laminectomia, devido à remoção dos processos articulares, ligamento amarelo e interespinhoso, acarreta perda de $75 \%$ de estabilidade vertebral (SMITH \& WALTER, 1988).

Visando diminuir as aderências fibróticas à dura-máter, aconselha-se exatamente como TREVOR et al. (1991) sugeriram para laminectomias profundas, que o enxerto de gordura deve ter largura suficiente para cobrir o defeito da laminectomia, mas espessura inferior a $5 \mathrm{~mm}$. Essa sugestão é reforçada pelo fato de que TUDURY et al. (1985) ao realizar laminectomia tipo Funkquist A, porém sem colocação de qualquer tipo de enxerto, não verificaram distúrbios neurológicos imediatos ou tardios. Muito cuidado deve ser tomado durante o fechamento da fáscia epiaxial para evitar forçar o enxerto de gordura e/ou a musculatura epiaxial para dentro do canal espinhal (TREVOR et al., 1991). A manutenção do ligamento supra-espinhal sugerido por TUDURY et al. (1985) não perturba a realização da laminectomia, porém pode contribuir à compressão medular imediata ao término da cirurgia.

Como foram encontradas diferenças altamente significativas nos cálculos estatísticos das relações III e IV, ficou provado que a cirurgia também ocasiona achatamento do canal vertebral. Talvez, por se ter trabalhado com uma amostra reduzida de animais, os cálculos estatísticos não foram significativos com relação ao achatamento das medulas espinhais. O estreitamento látero-lateral do espaço subaracnoídeo, presente nas mielografias dos cinco animais do grupo I, correspondente ao disco intervertebral $\mathrm{T}_{13}-\mathrm{L}_{1}$, foi decorrente do tracionamento dorsal da dura-máter pelas aderências fibróticas. Este estreitamento latero-lateral do espaço subaracnoídeo pode ter contribuído para a não significância dos achatamentos dorso ventrais das medulas espinhais, provocados pela membrana da laminectomia formada no defeito ósseo espinhal.

A alta incidência de cifose observada durante o pós-operatório, neste estudo, episódio também verificado por FIALHO \& BERNIS (1975), FUNKQUIST \& SCHANTZ (1962) e BIGNOZZI \& MASCAGNI (1958), parece estritamente relacionada à ausência de suporte dos processos articulares e à dor resultante da compressão da medula espinhal e raízes nervosas pelo canal espinhal estreitado, a qual é minimizada por tal postura (FUNKQUIST \& SCHANTZ, 1962; FIALHO \& BERNIS, 1975). No presente estudo, tal como nos de FUNKQUIST \& SCHANTZ (1962), os cães (no 5, 6 e 7) que tiveram maior perda motora também exibiram maior grau de deformação medular.
Diante dos resultados desta pesquisa, aconselha-se não realizar em cães acometidos de afecções medulares na junção toracolombar a seguinte conjuntura de procedimentos cirúrgicos: laminectomia do tipo Funkquist A com elevação transcirúrgica dos corpos vertebrais, colocação de gordura como coxim supramedular, manutenção do ligamento supramedular, sutura tensa da fáscia toracolombar e bandagem compressiva pós-operatória. Mas ainda, em frente à existência de técnicas que oferecem exposição e descompressão equivalentes, porém com efeitos secundários irrelevantes também não se aconselham esses procedimentos cirúrgicos.

\section{REFERÊNCIAS BIBLIOGRÁFICAS}

BIGNOZZI, L., MASCAGNI, M. Laminectomia decompressive per tumore osteogênico vertebrale in un cane. Atti Soc Ital Sci Vet, v.12, p.264-269, 1958

FIALHO, S.A.G., BERNIS, W.O. Laminectomia dorsal no cão. Arq Esc Vet UFMG, v.27, n.2, p. 105-117, 1975.

FUNKQUIST, B., SCHANTZ, B. Influence of extensive laminectomy on the shape of the spinal canal. Acta Orthop Scand, n.56, p.7-50, 1962.

FUNKQUIST, B. Thoraco-lumbar disk protrusion with severe cord compression in the dog. III. Treatment by decompressive laminectomy. Acta Vet Scand, v.3, p.1-25, 1962.

FUNKQUIST, B. Decompressive laminectomy in thoracolumbar disc protrusion with paraplegia in the dog. J Small Anim Pract, v.11, p.445-451, 1970.

GAMBARDELLA, P.C. Dorsal decompressive laminectomy for treatement of thoracolumbar disc disease in dogs: A retrospective study of 98 cases. Am Coll Vet Surg, v.9, n.1, p.24-26, 1980 .

HORNE, T.R.,POWERS, R.D., SWAIM, S.F. Dorsal laminectomy techniques in the dog. J Am Vet Med Assoc, v.171, n.8, p.742-749, 1977.

KELLER, J.T., DUNSKER, S.B., MCWHORTER, J.M., et al The fate of autogenous grafts to the spinal dura. In experimental study. J Neurocir, v.49, p.412-417, 1978.

LOROCCA, H., MACNAB, I. The laminectomy membrane. J Bone Jt Surg, v.56 B, n. 3, p.545-550, 1974.

SNEDECOR, G.W., COCKRAN, W.G. Statistical methods. 8. ed. Iowa : Iowa State University, 1989. 503p.

SMITH, G.K., WALTER, M.C. Spinal decompressive procedures and dorsal compartment injuries: Comparative biomechanical study in canine cadavers. Am J Vet Res, v.49, n.2, p.266-273, 1988.

SWAIM, S.F., VANDEVELDE, M. Clinical and histologic evaluation of bilateral hemilaminectomy and deep dorsal laminectomy for extensive spinal cord decompression in the dog. J Am Vet Med Assoc, v.170, n.4, p.407-413, 1974.

SWAIM, S.F. Vertebral and spinal cord surgery. In: OLIVER, J.E., HOERLEIN, B.F., MAYHEEW, I.G. Veterinary neurology. Philadelphia : Saunders, 1987. Cap.17., p.416-442. 
TREVOR, P.B., MARTIN, R.A., SAUNDERS, G.K., $\boldsymbol{e}$ t al Healing characteristics of free and pedicle fat grafts after dorsal laminectomy and durotomy in dogs. Vet Surg, v.20, n.5, p.282-290, 1991.

TROTTER, E.J., BRASMER, T.H., DE LAHUNTA, A Modified deep dorsal laminectomy in the dog. Cornell Vet., v. 65, p.402-427, 1975 .

TROTTER, E.J., CRISSMAN, J., ROBSON, D., et al. Influence of nonbiologic implants on laminectomy membrane formation in dogs. Am J Vet Res, v.49, n.5, p.634-642, 1988.
TROTTER, E.J. Dorsal laminectomy for treatment of thoracolumbar disk disease. In: BOJRAB, M.J., BIRCHARD, S.J., TOMLINSON, J.L. Current techniques in small Animal surgery. 3.ed. Philadelphia : Lea \& Febiger, 1990. Cap.43, p.608-621.

TUDURY, E.A., FIALHO, S.A.G., PAULO, N.M., et al. Avaliação de dois métodos para prevenção da cifose pósoperatória na laminectomia dorsal de cães. Rev Centro Ciências Rurais, v.15, n.1, 57-68, 1985.

YOVICH, J.C., READ, R., EGER, C. Modified lateral spinal decompression in $61 \mathrm{dogs}$ with thoracolumbar disc protrusion. J Small Anim Pract,. v.35, n.7, p.351-356, 1994

Ciência Rural, v. 31, n. 1, 2001. 